\title{
Diffusion on a Solid Surface: Anomalous is Normal
}

\author{
J. M. Sancho, ${ }^{1}$ A. M. Lacasta, ${ }^{2}$ K. Lindenberg, ${ }^{3}$ I. M. Sokolov, ${ }^{4}$ and A. H. Romero ${ }^{5}$ \\ ${ }^{1}$ Departament d'Estructura i Constituents de la Matèria, Facultat de Física, Universitat de Barcelona, \\ Diagonal 647, E-08028 Barcelona, Spain \\ ${ }^{2}$ Departament de Física Aplicada, Universitat Politècnica de Catalunya, Avinguda Doctor Marañon 44, E-08028 Barcelona, Spain \\ ${ }^{3}$ Department of Chemistry and Biochemistry 0340, and Institute for Nonlinear Science, University of California, \\ San Diego, La Jolla, California 92093-0340, USA \\ ${ }^{4}$ Institut für Physik, Humboldt Universitcet zu Berlin, Newtonstrasse 15, 12489 Berlin, Germany \\ ${ }^{5}$ Advanced Materials Department, IPICyT, Camino a la presa San José 2055, CP 78216, San Luis Potosí, San Luis Potosí, México
} (Received 22 October 2003; published 23 June 2004)

\begin{abstract}
We present a numerical study of classical particles diffusing on a solid surface. The particles' motion is modeled by an underdamped Langevin equation with ordinary thermal noise. The particle-surface interaction is described by a periodic or a random two-dimensional potential. The model leads to a rich variety of different transport regimes, some of which correspond to anomalous diffusion such as has recently been observed in experiments and Monte Carlo simulations. We show that this anomalous behavior is controlled by the friction coefficient and stress that it emerges naturally in a system described by ordinary canonical Maxwell-Boltzmann statistics.
\end{abstract}

PACS numbers: 05.40.-a, 68.35.Fx, 68.43.Jk

Diffusion of atoms, molecules, and clusters on solid surfaces occurs in a number of modern technologies involving self-assembled molecular film growth, catalysis, and surface-bound nanostructures [1]. The study of the motion of small and large organic molecules [2,3], and of adsorbed metal clusters composed of tens and even hundreds of atoms [4,5], has led to the unexpected observation that, as with single atoms [6], long jumps may play a dominant role in these motions.

Theoretical, numerical, and phenomenological discussions of surface diffusion have led to the clear understanding that jumps beyond nearest neighbors are ubiquitous in some parameter regimes [7]. However, these studies focus on the fact that the motion in periodic potentials is necessarily diffusive on very long time scales. In a random potential the motion at long times is either diffusive or, in the case of overdamped motion in some parameter regimes, subdiffusive. The latter case has been thoroughly studied [8]. Of interest to us, the possibility that jumps can be so long as to lead to superdiffusive motion over appreciable intermediate time scales, has been recognized as an interesting problem, but one in which Lévy walks or flights [9] are invoked as a model input. Lévy-walk-like behavior is clearly observed in Hamiltonian systems [10,11] and in microcanonical simulations $[11,12]$. We show that the behavior observed for small friction in the canonical case shares several features of the Lévy walk behavior in a Hamiltonian system; however, a closer look at the properties of this process shows that it is considerably different.

While a detailed analysis of surface diffusion requires extensive calculations (e.g., ab initio or molecular dynamics), even the most powerful currently available computers cannot carry such calculations to anywhere near experimentally relevant time scales [13]. Moreover, current experimental probes of the topography of surfaces, scanning tunneling microscopy and atomic force microscopy, are usually carried out at relatively high temperatures, which leads to additional difficulties for first-principles calculations. Therefore, simpler approaches are essential and valuable [7].

We consider a generic model of classical particles moving in a two-dimensional potential, under the action of thermal fluctuations and dissipation, the important control parameter being the friction coefficient. In spite of the simplicity of the model, we find that it reproduces the entire range of experimentally and computationally observed phenomena, from superdiffusion all the way to subdiffusion. The equation of motion of a particle of mass $m$ on the surface is

$$
m \ddot{\mathbf{x}}=-\nabla V(\mathbf{x} / \lambda)-\mu \dot{\mathbf{x}}+\boldsymbol{\xi}(t),
$$

where $\lambda$ is the characteristic length scale of the potential. The parameter $\mu$ is the coefficient of friction, and the $\xi_{i}(t)$ are mutually uncorrelated white noises that obey the fluctuation-dissipation relation $\left\langle\xi_{i}(t) \xi_{j}\left(t^{\prime}\right)\right\rangle=2 \mu k_{B} T \delta_{i j} \delta\left(t-t^{\prime}\right)$. We first consider the nonseparable periodic potential $V(x, y)=V_{0} \cos [\pi(x+$ $y) / \lambda] \cos [\pi(x-y) / \lambda]$, where $V_{0}$ is the barrier height at the saddle points. Equation (1) can be rewritten in scaled dimensionless variables, $r_{x}=x / \lambda, r_{y}=y / \lambda$, and $\tau=$ $\sqrt{V_{0} / m} t / \lambda$, leaving only two independent parameters, the scaled temperature $\mathcal{T}$, and the scaled dissipation $\gamma$,

$$
\mathcal{T}=k_{B} T / V_{0}, \quad \gamma=\mu \lambda / \sqrt{m V_{0}} .
$$

We study three properties of the motion of the particle: the mean square displacement, the dependence of the diffusion coefficient on the friction, and the probability density function of displacements. 


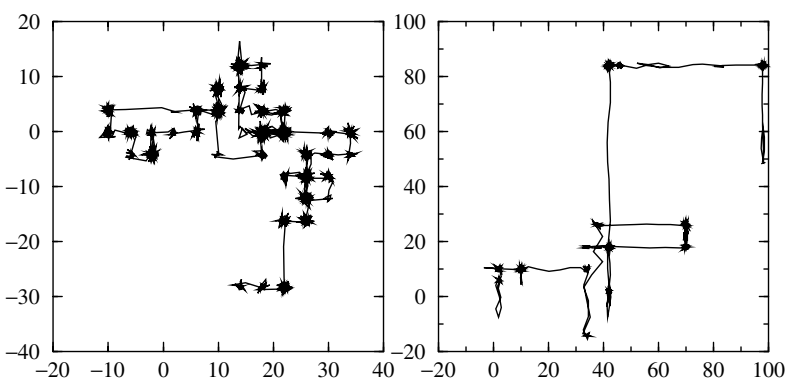

FIG. 1. Left: A trajectory for $\gamma=1$ over $t=20000$ time units. Right: A trajectory for $\gamma=0.04$ over $t=15000$ time units. The period of the potential is $\lambda=4$. Note the different scales in the two panels.

Normal diffusive behavior is characterized by a linear time dependence of the mean square displacement, $\left\langle r^{2}(\tau)\right\rangle \sim \tau$. The brackets denote an ensemble average over realizations of the noise. Nondiffusive behavior shows a different time dependence, $\left\langle r^{2}(\tau)\right\rangle \sim \tau^{\alpha}$, with $\alpha>1(<1)$ for superdiffusive (subdiffusive) motion. In Fig. 1 we show typical trajectory segments obtained upon numerical simulation of the equations of motion with $\mathcal{T}=0.2$ (we use this value throughout) for particles initially located at the center of the system. One trajectory (left panel) is for a large friction coefficient, and the particle follows typical diffusive motion characterized by short steps of length $\sim \lambda$ and frequent changes in direction. The other (right panel) corresponds to a small friction coefficient and clearly shows the preponderance of long $(\gg \lambda)$ tracks along Cartesian coordinates.

The evolution of $\left\langle r^{2}\right\rangle$ averaged over 5000 particles is shown in Fig. 2 for several friction coefficients. The particles are initially deposited in a square of side $2 \pi$ around the center of the system according to a Boltzmann equilibrium distribution for the positions and for the velocities. For very long times the motion is diffusive, as expected, but for small $\gamma$ and at intermediate times there is clear superdiffusive ballistic $(\alpha=2)$ behavior over several decades in time, reflective of the long straight stretches seen in the low- $\gamma$ trajectory in Fig. 1. We stress that this behavior has emerged naturally and has not required explicit implementation of any but ordinary thermal fluctuations.

Even though the motion of the particle may include long superdiffusive stretches, at long times the motion is necessarily diffusive. The dependence of the diffusion coefficient on the friction for small and for large $\gamma$ can be obtained analytically using the approximate relation $D \approx\left\langle l^{2}\right\rangle / 2 \tau_{0}$, where $\left\langle l^{2}\right\rangle$ is the mean square size of a jump out of one well and into another, and $\tau_{0}^{-1}$ is the mean jump rate (related to the familiar "mean escape rate"). In the overdamped regime, jumps typically occur from one well to a neighboring well, so $\left\langle l^{2}\right\rangle \approx 1$. Familiar Kramers formulas can be used to obtain the mean escape rate [14], with the result

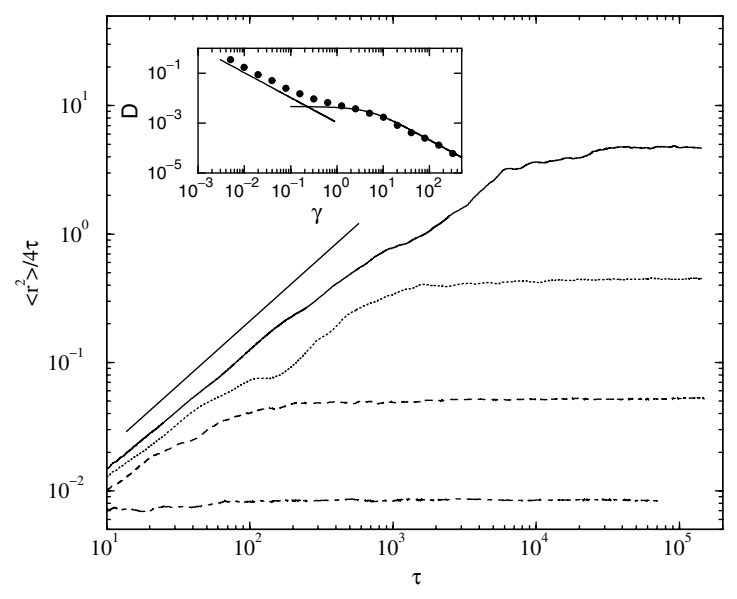

FIG. 2. $\left\langle r^{2}\right\rangle / 4 \tau$ for a particle in the periodic potential, for $\gamma=0.0004$ (solid line), 0.004 (dotted line), 0.04 (dashed line), and 0.4 (dot-dashed line). The straight-line segment has unit slope as a guide to the eye. Inset: Diffusion coefficient vs $\gamma$. The solid lines correspond to the theoretical calculations [Eqs. (3) and (4)].

$$
D \sim \frac{1}{2 \pi}\left(\sqrt{\frac{\gamma^{2}}{4}+2 \pi^{2}}-\frac{\gamma}{2}\right) e^{-1 / \mathcal{T}} \sim \frac{\pi}{\gamma} e^{-1 / \mathcal{T}} .
$$

The $\gamma^{-1}$ dependence of $D$ arises because $\left\langle l^{2}\right\rangle$ is independent of $\gamma$ while $\tau_{0} \sim \gamma$. In the underdamped limit a number of approximations can be invoked [15]: (a) Particles move preferentially along directions of lowest potential barriers, so that the motion is essentially one-dimensional between trapping events. (b) The particles must have initial energy above the barrier. As they move, they lose energy until they become trapped. (c) The slowest variable is the energy loss. An estimate of the time $\tau(E)$ it takes a particle to traverse the distance to a neighboring well can be obtained by assuming a constant energy. One finds $\tau(E) \approx(1 / \pi \sqrt{2}) \ln (16 / E)$ for $E$ close to the barrier. (d) One can separately estimate the energy actually lost due to slow dissipation in such a traversal. The result is $\Delta E \approx-\sqrt{8} \gamma \pi$. (e) The distance $l(E)$ traveled by a particle of initial energy $E$ before being trapped then is $l(E) \approx-(E / \Delta E) \approx \pi E / \sqrt{8} \gamma$. (f) The mean squared displacement is the average of $l^{2}(E)$ over the initial Boltzmann distribution of energies, which gives $\left\langle l^{2}\right\rangle=(\pi \mathcal{T} / 2 \gamma)^{2}$. Standard results [14] lead to $\tau_{0} \sim 1 / \gamma$ [16], but now $\left\langle l^{2}\right\rangle \sim \gamma^{-2}$ [15]. The result is

$$
D \sim \frac{\pi \mathcal{T}}{4 \gamma} e^{-1 / \mathcal{T}}
$$

that is, again an inverse dependence on friction. The theoretical diffusion coefficient as a function of the friction parameter is shown as the solid curves in the inset of Fig. 2. The symbols are the simulation results. The $\gamma^{-1}$ dependences have been noted in the literature [7], 
but we have provided explicit forms with no adjustable parameters.

The diffusion coefficient characterizes only the very long-time asymptotic dynamics. The interesting intermediate dynamics in the low friction regime that gives rise to long stretches of ballistic motion is reflected in the probability distribution function (PDF) $P(r, \tau)$ of particle displacements $r$ at time $\tau$. This PDF is shown in Fig. 3 for $\gamma=0.0004$ and four different time intervals $\tau$. For comparison, we also show a typical PDF for high damping $(\gamma=1)$ at the intermediate time interval. In the high- $\gamma$ curve the highest maximum corresponds to no jumps (by far the most likely event at short times). The next is associated with jumps to a nearest neighbor well, and so on. In contrast, the low- $\gamma$ curves show a very different behavior, with features strongly resembling those of a Lévy-walk model $[10,17]$ : a peak at small displacements, a power-law intermediate regime, and a side hump at high displacements. Each of these is a distinct signature of Lévy-walk-like dynamics, but one must be cautious in the detailed interpretation of these components. The persistent small displacement peak is associated with long trapping periods during which a particle does not move at all because its energy is not sufficient to overcome the barrier. The high displacement peak, which moves outward with velocity of order unity, is associated with the ballistic motion of those particles that acquire enough energy to move (and lose it very slowly). Genuine Lévywalk dynamics also exhibit a low displacement peak and a superdiffusive peak separated by a power-law behavior, but there are some important differences. First, our distribution reflects ballistic transport in the intermediate regime (in the language of Ref. [17], ballistic transport occurs when $0<\alpha<1$ in the Lévy model), whereas the regime where the Lévy model shows the features we have described is associated with sub-ballistic (but still superdiffusive) behavior (again, in the language of Ref. [17], the behavior when $1<\alpha<2$ ). Second, the slope in our

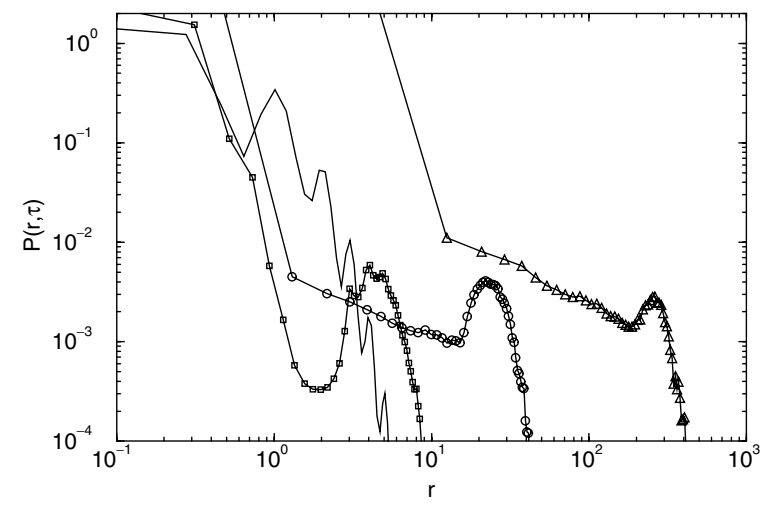

FIG. 3. Log-log plot of $P(r, \tau)$ for $\gamma=0.0004$ and three different values of time intervals: $\tau=20$ (squares), 100 (open circles), 1000 (triangles), and for $\gamma=1.0$ at the time interval $\tau=100$ (solid curve). power-law regime (approximately 0.7) is not related to the exponent $\alpha$ in the mean square displacement as it is for the Lévy walk (where the slope is $4-\alpha$ ). Third, our side hump is strongly broadened, whereas the side hump in the Lévy-walk model is associated with motion at a single constant velocity. In our case the velocity varies according to the equilibrium Maxwell-Boltzmann distribution. Nevertheless, the qualitative features of our distribution track those of the Lévy walk. Note that the existence of the pronounced side hump reflects the fact that the particles performing long steps ("flights") are those with a velocity in the tail of the Maxwellian distribution. This latter contribution to the PDF in a potential system arises from a small subset of particles and is thus to be distinguished from that of a typical underdamped free Brownian particle [18]. At long times, $\tau \gg 1 / \gamma$, both the long trapping and ballistic features are, of course, no longer present as diffusive motion dominates the behavior. Preliminary numerical analysis of the velocity power spectrum agrees with this scenario. Explicit results will appear in [15].

Disorder in surfaces occurs due to the presence of vacancies and other defects. As described in [8,19-21], we have generated a random potential surface described by a Gaussian distribution with a correlation function $\left\langle V(\mathbf{x}) V\left(\mathbf{x}^{\prime}\right)\right\rangle=\left(\varepsilon / 2 \pi \lambda^{\prime 2}\right) \exp \left(-\left|\mathbf{x}-\mathbf{x}^{\prime}\right|^{2} / 2 \lambda^{\prime 2}\right)$. We set the intensity $\varepsilon=100$ and the characteristic length $\lambda^{\prime}=$ 4 in our simulations.

The exponent $\alpha$ in $\left\langle r^{2}(\tau)\right\rangle \sim \tau^{\alpha}$ at intermediate times shows the entire range of behaviors from subdiffusive to superdiffusive with changing friction. In Fig. 4 we show $\left\langle r^{2}(\tau)\right\rangle / 4 \tau$, averaged over 5000 particles, as a function of time, for several values of $\gamma$. In the overdamped regime we clearly observe subdiffusive behavior $(\alpha<1)$ [8], while superdiffusive $(\alpha>1)$ behavior is seen for very

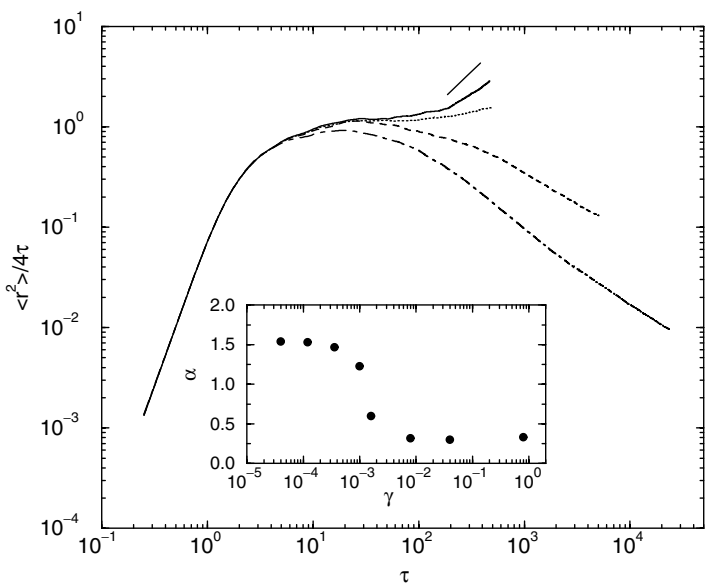

FIG. 4. $\left\langle r^{2}\right\rangle / 4 \tau$ for a particle in the random potential for $\gamma=$ 0.0001 (solid line), 0.001 (dotted line), 0.003 (dashed line), and 0.008 (dot-dashed line). The straight-line segment has unit slope as a guide to the eye. Inset: Exponents $\alpha$ versus friction coefficient $\gamma$. 
small $\gamma$. The exponents $\alpha$, calculated over the last decade of the time variation of the mean square displacement within our finite simulation times, are plotted in the inset of Fig. 4 as a function of $\gamma$. Although the subdiffusive behavior is probably the true asymptotic behavior in the overdamped case [8], further theoretical and numerical support are needed to assess whether superdiffusion is the asymptotic behavior in the underdamped case.

We summarize our findings. We have explored the behavior of a particle in a two-dimensional potential subject to thermal fluctuations described by ordinary Langevin dynamics. In a periodic potential, in the underdamped regime, the motion of the particle includes a ballistic range that can extend over many decades of time. The PDF of the particle's displacements under these conditions shows a structure strongly resembling one for Lévy walks. This may explain a number of observations involving superdiffusive motion of organic molecules [3] and atomic clusters [5] on surfaces without the need to invoke extraordinary fluctuations beyond the usual thermal description. The long-time behavior is diffusive in all cases, and we have been able to predict theoretically the dependence of the diffusion coefficient on friction over essentially the entire range of values of the friction parameter with no adjustable parameters. The situation in a random potential is even more complex, and exhibits a wide range of subdiffusive to superdiffusive regimes. Further analysis of the random potential case, and a more extensive presentation of the periodic problem, will be detailed elsewhere [15].

This work was supported by the MCyT (Spain) under Project No. BFM2003-07850, by the Engineering Research Program of the Office of Basic Energy Sciences at the U.S. Department of Energy under Grant No. DE-FG03-86ER13606, and by a grant from the University of California Institute for México and the United States (UC MEXUS) and the Consejo Nacional de Ciencia y Tecnología de México (CoNaCyT). A. H. R. acknowledges support from Millennium Initiative, Conacyt-Mexico, under Grant No. W-8001. I. M. S. acknowledges the hospitality of the University of Barcelona under the CEPBA grant, as well as partial financial support by the Fonds der Chemischen Industrie.

[1] G. E. Poirier and E. D. Plyant, Science 272, 1145 (1996); T. Yokoyama et al., Nature (London) 413, 619 (2001); K. Ho, J. Chem. Phys. 117, 11033 (2002); R. M. Tromp and J. B. Jannon, Surf. Rev. Lett. 9, 1565 (2002); T. AlaNissila, R. Ferrando, and S. C. Ying, Adv. Phys. 51, 949 (2002).

[2] J. Wecksesser et al., Surf. Sci. 431, 168 (1999); J. Weckesser, Ph.D. thesis, Swiss Federal Institute of Technology, Laussane, 2000.

[3] M. Schunack et al., Phys. Rev. Lett. 88, 156102 (2002).

[4] G. Ertl and H.-J. Freund, Phys. Today 52, No. 1, 32 (1999).

[5] W. D. Luedtke and U. Landman, Phys. Rev. Lett. 82, 3835 (1999).

[6] D. Cowell Senft and G. Ehrlich, Phys. Rev. Lett. 74, 294 (1995); T. R. Linderoth et al., Phys. Rev. Lett. 78, 4978 (1997); A. P. Graham et al., Phys. Rev. B 56, 10567 (1997); S-M Oh et al., Phys. Rev. Lett. 88, 236102 (2002).

[7] K. D. Dobbs and D. J. Doren, J. Chem. Phys.. 97, 3722 (1992); R. Ferrando, R. Spadacini, and G. E. Tommei, Phys. Rev. E 48, 2437 (1993); L. Y. Chen, M. R. Baldan, and S. C. Ying, Phys. Rev. B 54, 8856 (1996); J. L. Vega, R. Guantes, and S. Miret-Artés, Phys. Chem. Chem. Phys. 4, 4985 (2002); R. Guantes et al., J. Chem. Phys. 119, 2780 (2003); S. J. Mitchell, S. Wang, and P. A. Rikvold, Faraday Discuss. 121, 53 (2002).

[8] A. H. Romero and J. M. Sancho, Phys. Rev. E 58, 2833 (1998).

[9] M. F. Shlesinger, G. M. Zaslavsky, and J. Klafter, Nature (London) 363, 31 (1993).

[10] J. Klafter and G. Zumofen, Phys. Rev. E 49, 4873 (1994).

[11] J. L. Vega, R. Guantes, and S. Miret-Artés, J. Phys. Condens. Matter 14, 6193 (2002).

[12] R. Guantes, J. L. Vega, and S. Miret-Artés, Phys. Rev. B 64, 245415 (2001).

[13] A. Gross, Surf. Sci. Rep. 31, 235 (1998).

[14] P. Hänggi, P. Talkner, and M. Berkovec, Rev. Mod. Phys. 62, 251 (1990).

[15] A. M. Lacasta et al. (to be published).

[16] J. M. Sancho, A. H. Romero, and K. Lindenberg, J. Chem. Phys. 109, 9888 (1998).

[17] J. Klafter and G. Zumofen, Physica (Amsterdam) 196A, 102 (1993).

[18] P. M. Chaikin and T. C. Lubensky, Principles of Condensed Matter Physics (Cambridge University Press, Cambridge, 1995), Sec. 7.5.

[19] J. García-Ojalvo and J. M. Sancho, Noise in Spatially Extended Systems (Springer, New York, 1999).

[20] H. A. Makse, S. Havlin, M. Schwartz, and H. E. Stanley, Phys. Rev. E 53, 5445 (1996);E. Koscielny-Bunde, A. Bunde, S. Havlin, E. Roman, Y. Goldreich, and H. J. Schellnhuber, Phys. Rev. Lett. 81, 729 (1998).

[21] A. H. Romero and J. M. Sancho, J. Comput. Phys. 156, 1 (1999); A. H. Romero, J. M. Sancho, and K. Lindenberg, Fluct. Noise Lett. 2, L79 (2002). 